\title{
Universiteit
}

Leiden

The Netherlands

\section{Superconductivity in a molecular metal cluster compound}

Bakharev, O.; Bono, D.H.F.; Brom, H.B.; Schnepf, A.; Schnöckel, H.; Jongh, L.J. de

\section{Citation}

Bakharev, O., Bono, D. H. F., Brom, H. B., Schnepf, A., Schnöckel, H., \& Jongh, L. J. de. (2006). Superconductivity in a molecular metal cluster compound. Physical Review Letters, 96(11), 117002. doi:10.1103/PhysRevLett.96.117002

Version: $\quad$ Not Applicable (or Unknown)

License: $\quad$ Leiden University Non-exclusive license

Downloaded from: https://hdl.handle.net/1887/73929

Note: To cite this publication please use the final published version (if applicable). 


\title{
Superconductivity in a Molecular Metal Cluster Compound
}

\author{
O. N. Bakharev, ${ }^{1, *}$ D. Bono, ${ }^{1}$ H. B. Brom, ${ }^{1}$ A. Schnepf, ${ }^{2}$ H. Schnöckel, ${ }^{2}$ and L. J. de Jongh ${ }^{1}$ \\ ${ }^{1}$ Kamerlingh Onnes Laboratory, Leiden University, P.O. Box 9504, 2300RA Leiden, The Netherlands \\ ${ }^{2}$ Institut für Anorganische Chemie, Universität Karlsruhe, 76128 Karlsruhe, Germany \\ (Received 24 November 2005; published 21 March 2006)
}

\begin{abstract}
Compelling evidence for band-type conductivity and even bulk superconductivity below $T_{\mathrm{c}} \approx 8 \mathrm{~K}$ has been found in ${ }^{69,71} \mathrm{Ga}$ NMR experiments in crystalline ordered, giant $\mathrm{Ga}_{84}$ cluster compounds. This material appears to represent the first realization of a theoretical model proposed by Friedel in 1992 for superconductivity in ordered arrays of weakly coupled, identical metal nanoparticles.
\end{abstract}

PACS numbers: 74.78.Na, 74.70.-b, 76.60.-k

In recent years it has become apparent that the chemical (bottom-up) route to nanostructures can be quite successful. Molecular metal cluster compounds form an excellent example in this respect [1]. These stoichiometric compounds form macromolecular solids, in which the cores of the macromolecules can be seen as metal nanoparticles. Often the cluster molecules are ionic and, together with suitable counterions, form crystalline 3D lattices. These "self-organized nanostructures" can thus be viewed as 3Dordered arrays of identical metal nanoparticles, embedded in the dielectric matrix formed by ligand molecules plus counterions. Until recently, in all compounds studied so far electron transfer between clusters proved negligible, the materials being electrically insulating [2,3]. Accordingly, the experiments were probing single-particle properties at the nanoscale, such as surface effects, quantum-size effects, and the size-induced metal-nonmetal transition [4].

However, given the strong similarity of metal cluster compounds to, e.g., the (alkali-doped) fullerenes $\left(\mathrm{C}_{60}\right)$, it was expected [2] that by doping or introducing mixed valency a novel class of materials showing (super)conductivity could be obtained. Indeed, a few years ago, the mixed-valent "giant" $\mathrm{Ga}_{84}$ cluster compound $\mathrm{Ga}_{84}\left[\mathrm{~N}\left(\mathrm{SiMe}_{3}\right)_{2}\right]_{20}-\mathrm{Li}_{6} \mathrm{Br}_{2}(\mathrm{thf})_{20} \cdot 2$ toluene was synthesized [5], its crystal and molecular structure being determined by $x$-ray diffraction [6]. The $\left[\mathrm{Ga}_{84} \mathrm{R}_{20}\right]^{4-}$ molecules (called $\mathrm{Ga}_{84}^{4-}$ in what follows) form a fully ordered ionic crystal together with the counterions $\left(2\left[\mathrm{Li}(\mathrm{thf})_{4}\right]^{+}\right.$and $\left.2\left[\mathrm{Li}_{2} \mathrm{Br}(\mathrm{thf})_{6}\right]^{+}\right)$. The mixed-valent property arises since the ion $\left[\mathrm{Ga}_{84} \mathrm{R}_{20}\right]^{3-}\left(\mathrm{Ga}_{84}^{3-}\right)$ also exists [6], having the same molecular structure but different crystalline packing of the cluster molecules [6]. Since synthetic conditions for both moieties are different, samples obtained are "crystallographically pure," meaning that by $\mathrm{x}$ ray no trace of the other moiety can be detected. However, as evidenced by EPR, a small amount (1\%) of mixed valency is still present, probably due to local departures from stoichiometry in the concentration of counterions.

In earlier resistivity and magnetization experiments on $\mathrm{Ga}_{84}^{4-}$ samples [7], indications were already found for metallic behavior, and even superconductivity below a transition temperature $T_{\mathrm{c}} \approx 7.2 \mathrm{~K}$, much higher than $T_{\mathrm{c}} \approx$
1.1 K known for bulk $\alpha$-Ga metal. The observed superconducting (SC) fraction was only $0.01 \%$, however, and, since it is known that $\mathrm{Ga}$ metal in confined geometries tends to have higher $T_{\mathrm{c}}$ values, even as high as $6 \mathrm{~K}$ [8], these data were interpreted with caution and an explanation in terms of bulk metal inclusions, originating from possible deterioration and subsequent coalescence of clusters, could not be ruled out [9]. Additional experiments were needed therefore to provide unambiguous proof for the occurrence of bulk superconductivity related to weak intermolecular charge transfer, similar as in doped $\mathrm{C}_{60}$ or other molecular (super)conductors. Such proof is presented here on basis of ${ }^{69,71}$ Ga NMR experiments in both the metallic conducting and the SC phases of these cluster solids.

NMR is an element-specific probe that can evidence two hallmarks of metallic conductivity. First, the magnetic hyperfine interaction between nuclear moments and conduction electron spins produces a shift $\delta \nu$ of the NMR frequency $\nu_{0}$. This Knight shift, $K_{S}=\delta \nu / \nu_{0}$, is proportional to the density of states at the Fermi-energy, $D\left(E_{F}\right)$. Second, this interaction leads to a linear $T$ dependence (Korringa law) of the nuclear spin-lattice relaxation rate, $T_{1}^{-1} \propto a T$. The proportionality constant is $a=K_{S}^{2} / S$, where $S$ is the Korringa constant. For free $s$ electrons, $S$ is given theoretically by $\mu_{B}^{2} / \pi \hbar k_{B} \gamma^{2} \approx 2.826 \times 10^{-6} \mathrm{~s} \mathrm{~K}$ for ${ }^{71} \mathrm{Ga}$ ( $\gamma$ denotes the nuclear gyromagnetic ratio). Moreover, NMR can also probe the superconductivity: When the conduction electrons below $T_{\mathrm{c}}$ become spin paired, both $T_{1}^{-1}$ and $K_{S}$ should decrease. Experimental details about the ${ }^{69,71} \mathrm{Ga}$ NMR technique can be found in a previous brief note [10] on preliminary results for $\mathrm{Ga}_{84}^{4-}$ samples in the metallic conducting region $(T>10 \mathrm{~K})$. Meanwhile, several samples of both moieties have been studied and we present here representative results on three samples, labeled S1 $\left(\mathrm{Ga}_{84}^{4-}\right)$, S2 $\left(\mathrm{Ga}_{84}^{3-}\right)$, and S3 $\left(\mathrm{Ga}_{84}^{4-}\right)$. High- $T$ $(\sim 200 \mathrm{~K}){ }^{71} \mathrm{Ga}$ spectra are shown in Fig. 1 and display two main lines, one being unshifted and the other shifted by $0.4-0.5 \mathrm{MHz}$. The relative weight of these lines is sample dependent. Calibration of the Ga-NMR signals with an $\mathrm{Al}$ reference confirms, however, that the observed concentration of Ga nuclei in the combined spectra is as expected from the chemical formulas of the cluster compounds. 


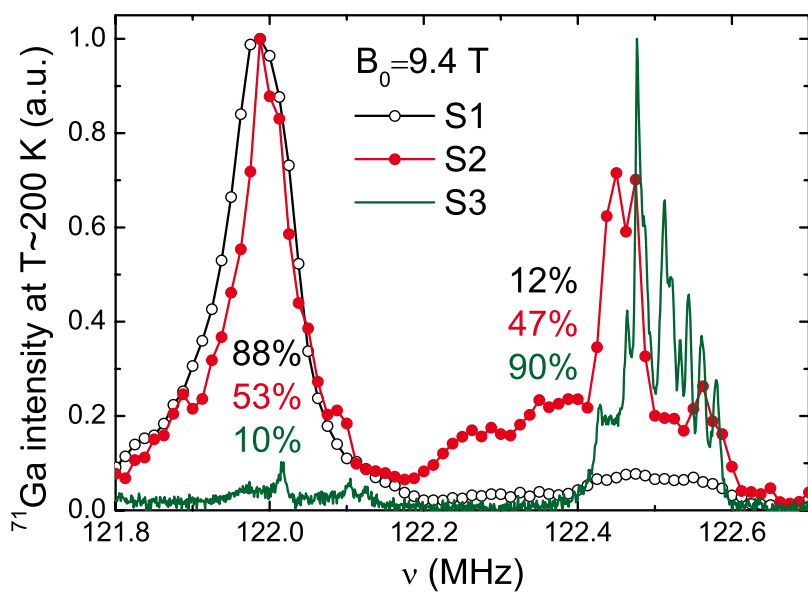

FIG. 1 (color online). High- $T$ spectra for $\mathrm{S} 1, \mathrm{~S} 2$, and S3, corresponding to an unshifted reference frequency $\nu_{0} \approx$ $122 \mathrm{MHz}\left(B_{0} \approx 9.4 \mathrm{~T}\right)$. They show two main lines, with varying relative weights.

Representative $T_{1}^{-1}$ data, measured on the maximum of the lines, are given in Fig. 2. The solid symbols refer to the unshifted line. The relaxation rate of these Ga nuclei is 1 to 2 orders of magnitude smaller than in $\alpha$-Ga metal [11] and does not follow the Korringa law. Hence, they lack the relaxation channel provided by conduction electron spins and only couple energetically to the phonons by quadrupolar relaxation, as proved by the measured ratio $\left.{ }^{69} T_{1} /{ }^{71} T_{1} \approx\left({ }^{71} Q /{ }^{69} Q\right)\right)^{2} \approx 0.4$ ( $Q$ denotes the nuclear quadrupolar moment). The unshifted line is therefore ascribed to a "nonconducting" (NC) fraction of the sample. By contrast, the points measured on the shifted line (open symbols) obey very well the Korringa law $T_{1}^{-1} \propto a T$ in a

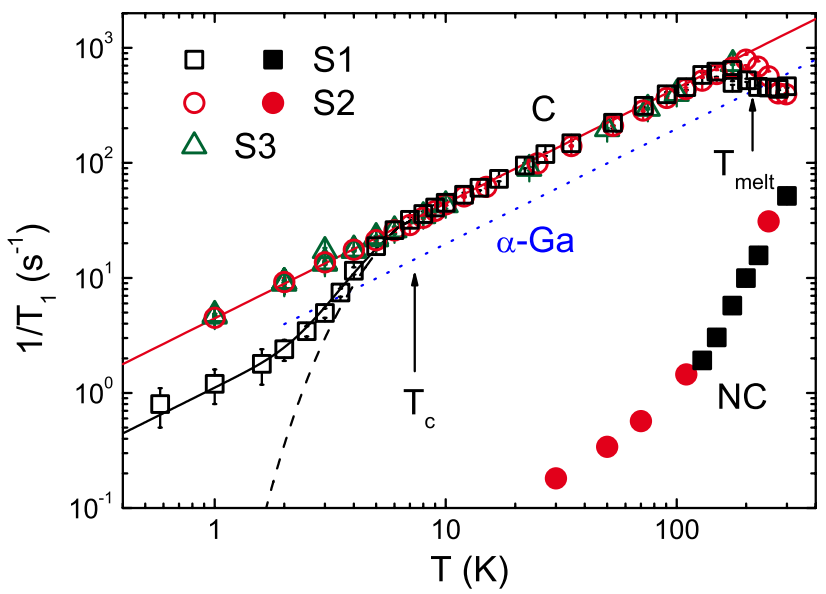

FIG. 2 (color online). $\quad T$ dependence of the ${ }^{71} \mathrm{Ga}-T_{1}^{-1}$ measured on the maxima of the two lines displayed in Fig. 1. The open symbols follow the Korringa law when $T \geq T_{\mathrm{c}}$ (and $B_{0} \geq B_{\mathrm{c} 2}$ ), and refer to the $C$ fraction (several fields $1.5 \mathrm{~T} \lesssim B_{0} \leq 9.4 \mathrm{~T}$ yield the same Korringa constant). The SC transition at $T_{\mathrm{c}} \approx$ $7.5 \mathrm{~K}$ is observed in $\mathrm{S} 1$ in $B_{0} \approx 2.39 \mathrm{~T}$. The dotted curve indicates the behavior of bulk $\alpha$-Ga. The other curves are fits (see text). Solid symbols refer to the unshifted line (NC fraction). wide $T$ range (below $T \sim 200 \mathrm{~K}$ down to $T_{\mathrm{c}}$ ), with $a=$ $4.45(20) \mathrm{s}^{-1} \mathrm{~K}^{-1}$ for all samples (i.e., independent of the $\mathrm{NC}$ fraction). This line can thus be attributed to a conducting (C) phase of the sample.

The origin of the NC fraction turns out to be a lack of toluene molecules, normally present as a crystal solvent and apparently essential to produce an ordered 3D packing of the cluster molecules. We found that when the crystals are taken out of the toluene solution, toluene molecules rapidly diffuse out of the sample which thereby loses its perfect crystalline order, with accompanying loss of conducting properties. Indeed, as is well known, in case of a small (intermolecular) charge transfer, band conductivity can be rapidly destroyed by even a small amount of disorder (Anderson localization). In the investigated samples the NC fraction varies from $88 \%$ down to $10 \%$ for the "purest" sample (S3, kept continuously in toluene liquid). Importantly, independent of the $\mathrm{C} / \mathrm{NC}$ ratio, we find always the same types of behavior of $T_{1}$ for the NC fractions, as well as for the $\mathrm{C}$ fractions in the metallic conducting region $(T>8 \mathrm{~K})$. This is a strong indication that the two phases exist separately; i.e., they are not mixed on a molecular level in the material. Most likely the NC phase is predominantly present at the surfaces of the crystallites, where toluene as crystal solvent is most readily removed. In the following, we only discuss the $\mathrm{C}$ fractions of the samples.

Interestingly, the value $a$ found for the $\mathrm{C}$ fraction is about 2.3 times that of bulk $\alpha$-Ga metal [11], indicating a larger $D\left(E_{F}\right)$ for the cluster compound. The value found for the ratio of the ${ }^{71} \mathrm{Ga}$ and ${ }^{69} \mathrm{Ga}$ rates, ${ }^{69} T_{1} /{ }^{71} T_{1} \approx$ $\left({ }^{71} \gamma /{ }^{69} \gamma\right)^{2} \approx 1.6$, confirms the magnetic origin (the coupling to the electron spin) of the nuclear relaxation. The ${ }^{71} \mathrm{Ga}$ Knight shift is ${ }^{71} K_{S}=0.39(3) \%$ for this line, using for calibration the metallic ${ }^{63} \mathrm{Cu}$ reference signal $\left({ }^{63} K_{S}=\right.$ $0.238 \%$ ). We derive the Korringa constant, $S=K_{S}^{2} / a=$ $3.4(6) \times 10^{-6} \mathrm{~s} \mathrm{~K}$. This is only $\sim 15 \%$ higher than the theoretical (free-electron) value, indicating a predominantly $s$-like character of the itinerant electron density, with weak correlations. For comparison, for bulk $\alpha$-Ga the shift is ${ }^{71} K_{S} \approx 0.16 \%$, consistent with a smaller $D\left(E_{F}\right)$, and the resulting Korringa constant is $\sim 60 \%$ smaller than for the free-electron case. A more accurate method to evaluate $S$ is presented in Fig. 3, showing for several temperatures the variation of $T_{1} T$ over the $\mathrm{C}$ line in the ${ }^{71} \mathrm{Ga}$ spectrum for the (purest) sample S3. Despite the complexity of the spectrum (see below), all data points are in excellent agreement with metallic behavior with the same Korringa constant. A fit $\left(T_{1} T\right)^{-1}=\frac{\left(\nu-\nu_{0}\right)^{2}}{S \nu_{0}^{2}}$ is well obeyed with $S=3.85(9) \times 10^{-6} \mathrm{~s} \mathrm{~K}$. This is in good agreement with the former result based on measurements of the $T$ dependence of $T_{1}$ on the maximum of the line.

Another interesting feature is the strong effect of motional narrowing observed in the $T$ dependence of the spectra, also shown in Fig. 3. The spectrum at low $T$ is $\sim 200 \mathrm{kHz}$ wide, with a fine structure that we attribute to the different Ga sites (with different interatomic distances) 


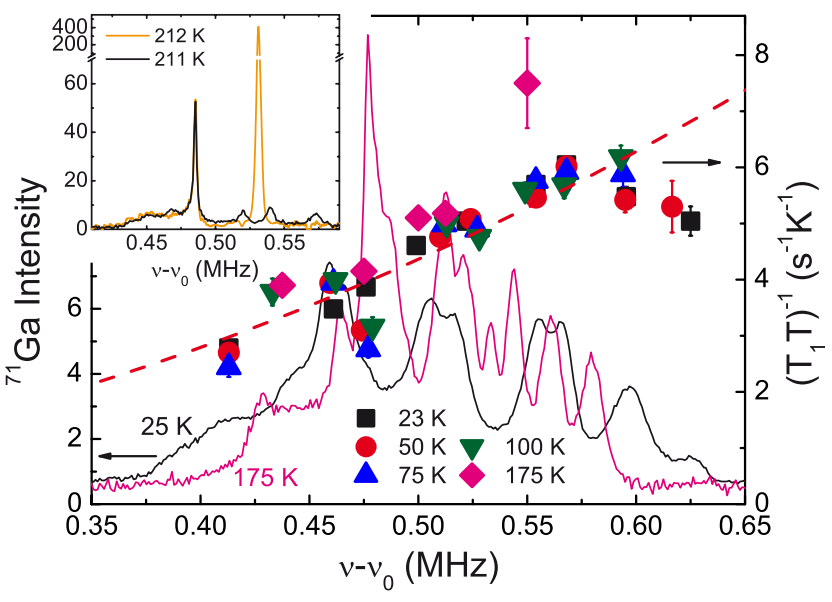

FIG. 3 (color online). Left axis (lines) and inset: $T$ dependence of the ${ }^{71} \mathrm{Ga}$ spectrum of the $\mathrm{C}$ fraction in $\mathrm{S} 3\left(\nu_{0} \approx 122 \mathrm{MHz}\right)$. The motional narrowing of the lines is very pronounced above $T_{\text {melt }} \approx 212 \mathrm{~K}$ (inset) and is more gradual below. Right axis (solid symbols): frequency dependence of $\left(T_{1} T\right)^{-1}$ over the ${ }^{71} \mathrm{Ga}$ spectrum in S3, for $T_{\mathrm{c}}<T<T_{\text {melt }}$. The dashed line is a fit (see text).

in the cluster cores [5,6]. For $\mathrm{S} 3$ (highest $\mathrm{C}$ fraction), the spectrum can be decomposed into at least 10 different lines. Within a very narrow range of temperature $(\sim 1 \mathrm{~K})$, the whole spectrum collapses above $T_{\text {melt }} \approx 212 \mathrm{~K}$ into two narrow lines, at 122.486 and $122.533 \mathrm{MHz}$ [Fig. 3 (inset)], the upper one having a linewidth at room temperature of only $1 \mathrm{kHz}$ in a field of $9.4 \mathrm{~T}$. The motional rate estimated from the linewidths increases from $0.4 \mathrm{MHz}$ around $T_{\text {melt }}$ to $10 \mathrm{MHz}$ at room temperature. Moreover, this line contains $\sim 70 \%$ of the Ga nuclei. Considering that $\sim 25 \%$ of the $\mathrm{Ga}$ atoms form the nearest-neighbor shell around the central $\mathrm{Ga}_{2}$ dimer [5,6], and that $\mathrm{x}$-ray studies show the freezing of rotations of the $\mathrm{Ga}_{2}$ dimer within this cage in this $T$ range, we associate the lower frequency line to the nearest-neighbor sites of the central dimer. These would be most affected by the dimer motions, explaining the weak motional narrowing observed even below $T_{\text {melt }}$ in particular for this subspectrum (Fig. 3). As seen in Fig. 2, the transition at $T_{\text {melt }}$ is accompanied by a drop (factor 3 ) in $T_{1}^{-1}$ of the $\mathrm{C}$ fraction. Apparently, the onset of the internal motions affects the $D\left(E_{F}\right)$ value in some as yet unknown way.

The combination of all the above results leaves no doubt that the observed spectra are due to Ga nuclei belonging to the cluster molecules and not to inclusions or particles of bulk Ga metal. The $T$ dependence of $T_{1}^{-1}$ is typical for nuclear relaxation via conduction electrons. The fact that the conductivity is lost when the ordered packing of the clusters is disturbed proves that it arises from intercluster charge transfer.

Turning next to the SC properties, we remark that, for all samples, magnetization data show the occurrence of bulk superconductivity of type II, with $T_{\mathrm{c}} \approx 7.4,7.8$, and $7.95 \mathrm{~K}$ for S1, S2, and S3, and a lower critical field $B_{\mathrm{c} 1}$ of the order of a few $0.01 \mathrm{~T}$. The upper critical field $B_{\mathrm{c} 2}$ appears to vary strongly, between $\sim 0.3 \mathrm{~T}$ (S2 and S3) up to a few Tesla (S1), i.e., (very) much lower than the value of $13.8 \mathrm{~T}$ reported in [7]. In fact, these low values for $B_{\mathrm{c} 2}$ are the reason why by NMR we could detect superconductivity only in samples with sufficiently high $B_{\mathrm{c} 2}$, since sensitivity requirements limited our NMR experiments to $B_{0} \geqslant 2 \mathrm{~T}$. As an example, the $T$ and field dependencies of $T_{1}^{-1}$ and $K_{S}$ are shown in Figs. 2 and 4 for the $\mathrm{C}$ fraction of $\mathrm{S} 1$ for $T \lesssim$ $10 \mathrm{~K}$. They provide definite proof for the occurrence of bulk superconductivity below $\approx 7.5 \mathrm{~K}$ for this sample, up to several Tesla. $K_{S}(T)$ can be well fitted with the prediction from the BCS model with $s$-wave symmetry and weak electron-phonon coupling, implying a SC gap $\Delta / k_{B}=$ $1.76 T_{\mathrm{c}} \sim 13 \mathrm{~K}$. Several other samples showed similar dependencies of the shift. The data in Fig. 4 do not allow one to distinguish between the predictions for the "clean" and "dirty" limits for the weak coupling model, but the strong coupling case is clearly excluded. We note that in Fig. 2 we do not observe a coherence peak in the $T$ dependence of $T_{1}^{-1}$ around $T_{\mathrm{c}}$. As recently discussed in [12], a strongly reduced coherence peak in $T_{1}^{-1}$, in combination with a gapped behavior (sharp drop) at a lower temperature than $T_{\mathrm{c}}$, is typical for an $s$-wave superconductor. As shown by the continuous curve in Fig. 2, our $T_{1}$ data can indeed be fitted, for $T \lesssim 0.85 T_{\mathrm{c}}$ by the sum of an exponential [ $\propto$ $\exp \left(-\Delta / k_{B} T\right)$, dashed curve] with $\Delta / k_{B}=13 \pm 2 \mathrm{~K}$ and a linear term, accounting for the relaxation of the normal electrons in the vortex cores [12].

In view of the strong effect of the toluene molecules on the conductivity, we attribute the strong sample dependence of $B_{\mathrm{c} 2}$ to a varying degree of lattice defects, related to missing toluene molecules in the otherwise ordered lattices of the conducting $\mathrm{Ga}_{84}$ phases. We recall that theoretical models for dirty superconductors predict a $B_{\mathrm{c} 2}$

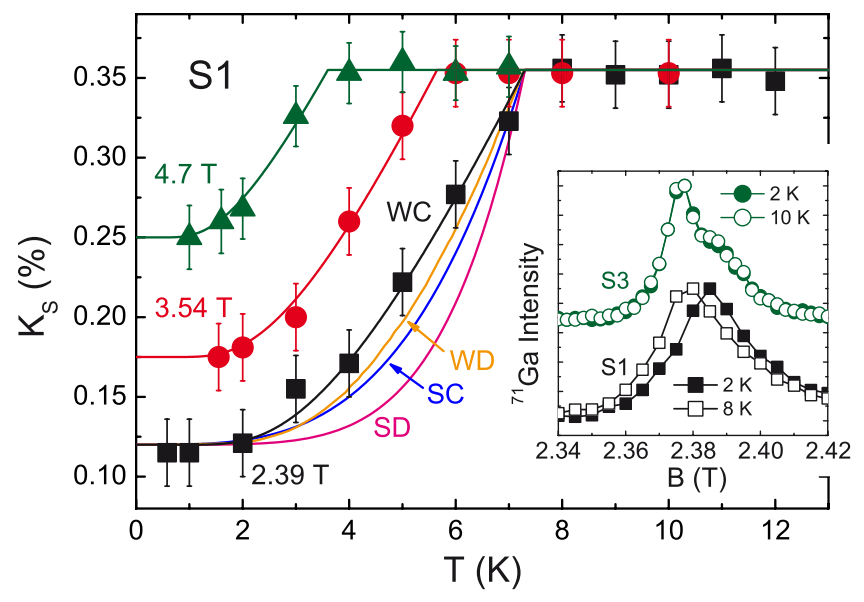

FIG. 4 (color online). $T$ and field dependences of the Knight shift $K_{S}$ for S1. Data for lowest field are compared with BCS theory for weak and strong coupling (symbols $\mathrm{W}$ and $\mathrm{S}$ ) in the clean and dirty limits (symbols C and D). The WC case is included for the other fields. Inset: ${ }^{71} \mathrm{Ga}$ spectra at low $T$. No shift is found for S3, having $B_{\mathrm{c} 2} \sim 0.25 \mathrm{~T} \ll B_{0} \approx 2.39 \mathrm{~T}$. 
inversely proportional to the electronic mean free path [13], whereas the values of $T_{\mathrm{c}}$ and the SC energy gap $\Delta$ should not be affected much by even high concentrations of defects (provided these are nonmagnetic), as observed. Although we expect the $\mathrm{NC}$ fractions to be present as a separate (surface) phase, a higher fraction of the (noncrystalline) NC phase may well entail a higher amount of lattice defects in the (crystalline) $\mathrm{C}$ phase of the sample. Indeed, S1 had the highest amount $(\sim 88 \%)$ of the NC phase [14]. We expect the sample studied in [7], with a reported $B_{\mathrm{c} 2} \approx 13.8 \mathrm{~T}$, to have contained an even larger concentration of defects. As a check on this interpretation, muon spin relaxation experiments were performed at the PSI facility in Switzerland on S3 in fields from zero to $0.3 \mathrm{~T}$ and $T>2 \mathrm{~K}$. The results will be reported elsewhere [15], but we mention here that also these data clearly prove the presence of bulk superconductivity with $B_{\mathrm{c} 2} \sim 0.25 \mathrm{~T}$ and $B_{\mathrm{c} 1} \sim 50 \mathrm{mT}$. Using theoretical expressions for the critical fields of a type II superconductor $[13,16]$, one obtains $\lambda \approx 80 \mathrm{~nm}$ and $\xi \approx 40 \mathrm{~nm}$ for the London penetration depth and the SC coherence length. Both values are much larger than the cluster core $(\varnothing \approx 1.4 \mathrm{~nm})$ and the average distance between the core centers $(\approx 2.3 \mathrm{~nm})$, in agreement with bulk superconductivity for the crystalline array of clusters. The quotient $\kappa=\lambda / \xi \approx 2$ indicates superconductivity of type II.

Summarizing, the above experiments provide compelling evidence for a band-type conductivity by weak intercluster charge transfer. How this charge transfer process occurs and via which intermediates is at present unknown, but it is clearly very sensitive to small local changes in the intercluster packing. This is reminiscent of the orientational disorder effects in $\mathrm{C}_{60}$. Comparing the molecular structure with that of $\mathrm{C}_{60}$, the intercluster transfer integral (i.e., the bandwidth) is expected to be much smaller, of order $t \sim 1-10 \mathrm{meV}$, considering that the $\mathrm{Ga}_{84}$ cluster cores are separated by surrounding ligand shells. On the other hand, the on-site Coulomb interactions will also be smaller in view of the larger cluster size. This may explain the - at first sight surprising - result that we find no evidence for strong electron correlation effects, in spite of the very narrow bandwidth.

Our findings present several important challenges to theory, such as the occurrence of bulk superconductivity with relatively high $T_{\mathrm{c}}$ at such small $t$ values, and the nature of the SC pairing mechanism. Assuming the latter to be phonon mediated, the $\mathrm{Ga}_{84}$ compound appears an almost perfect first experimental realization of the theoretical model advanced by Friedel [17] shortly after the discovery of superconductivity in fullerenes. He showed that for a crystalline ordered array of identical metal nanoclusters even a weak intercluster charge transfer can yield a large $T_{\mathrm{c}}$, provided the degeneracy of molecular levels near $E_{F}$ is sufficiently large. In this respect we mention that a larger value for $D\left(E_{F}\right)$ than for $\alpha$-Ga was obtained for the $\mathrm{Ga}_{84}$ compound from density functional calculations [18].
Quite recently, the same idea of obtaining a high $T_{\mathrm{c}}$ by increasing the $D\left(E_{F}\right)$ of the molecular-level-derived band through reduction of the transfer integral has been proposed [19] for a novel hybrid compound, which would consist of $\mathrm{C}_{60}$ molecules embedded in a metal-organic framework serving to keep them at a larger (tunable) distance.

This work is part of the research program of the "Stichting FOM" and is partially funded by the EC-RTN "QuEMolNa" (No. MRTN-CT-2003-504880), the ECNetwork of Excellence "MAGMANet" (No. 515767-2), and the DFG-Centre of Functional Nanostructures (Karlsruhe).

*Presently at Laboratory for Biomolecular NMR Spectroscopy, Department of Chemistry, University of Aarhus, Denmark.

[1] G. Schmid, J. Chem. Soc. Dalton Trans. 7, 1077 (1998); G. Schmid and L.F. Chi, Adv. Mater. 10, 515 (1998); A. Ecker, E. Weckert, and H. Schnöckel, Nature (London) 387, 379 (1997); A. Schnepf and H. Schnöckel, Angew. Chem., Int. Ed. 41, 3532 (2002); Metal Clusters in Chemistry, edited by P. Braunstein, L. A. Oro, and P. Raithby (Wiley-VCH and Weinheim, New York, 1999), Vols. I-III.

[2] Physics and Chemistry of Metal Cluster Compounds, edited by L. J. de Jongh (Kluwer, Dordrecht, 1994).

[3] J. A. Reedijk et al., Phys. Rev. B 57, R15116 (1998).

[4] F. M. Mulder et al., Nature (London) 367, 716 (1994); Y. Volokitin et al., Nature (London) 384, 621 (1996); F. C. Fritschij, H. B. Brom, L. J. de Jongh, and G. Schmid, Phys. Rev. Lett. 82, 2167 (1999); P. M. Paulus et al., Phys. Rev. B 64, 205418 (2001).

[5] A. Schnepf and H. Schnöckel, Angew. Chem., Int. Ed. 40, 711 (2001).

[6] A. Schnepf et al., Inorg. Chem. 42, 7731 (2003).

[7] J. Hagel et al., J. Low Temp. Phys. 129, 133 (2002).

[8] E. V. Charnaya et al., Phys. Rev. B 58, 467 (1998).

[9] The $\mathrm{Ga}_{84}$ compounds are highly air sensitive.

[10] O. N. Bakharev et al., Eur. Phys. J. D 24, 101 (2003). Unfortunately, a misprint occurred in this paper: all $T_{1}^{-1}$ values should be multiplied by $\sim 7.4$.

[11] R. H. Hammond, E. G. Wikner, and G. M. Kelly, Phys. Rev. 143, 275 (1966).

[12] K. Magishi et al., Phys. Rev. B 71, 024524 (2005).

[13] M. Tinkham, Introduction to Superconductivity (McGrawHill, New York, 1975).

[14] It was studied before the problem of the missing toluene molecules was realized, and had been taken out of the toluene solution.

[15] D. Bono et al. (to be published).

[16] E. H. Brandt, Phys. Rev. B 68, 054506 (2003).

[17] J. Friedel, J. Phys. II (France) 2, 959 (1992).

[18] J. Frenzel, S. Gemming, and G. Seifert, Phys. Rev. B 70, 235404 (2004).

[19] S. Hamel, V. Timoshevskii, and M. Côte, Phys. Rev. Lett. 95, 146403 (2005). 